of predicted remains quite constant at around $70 \%$. There is an increase in recording of co-morbidities from around $5 \%$ in young patients to around $20 \%$ in the very elderly. There is a clear and dramatic fall in a number of measures of process and treatment such as histological confirmation rate, anti-cancer treatment, and chemotherapy use beginning around the 7 th decade of life. The histological confirmation rate falls from $75 \%$ at age 65 to $40 \%$ at age 85 - the corresponding rates for specific anti-cancer therapy are $65 \%$ and $25 \%$. More concerning is the finding that these variations persist when corrected for PS and co-morbidities (see Abstract P215 Figure 1 ), with treatment rates falling from $50 \%$ to $4 \%$.

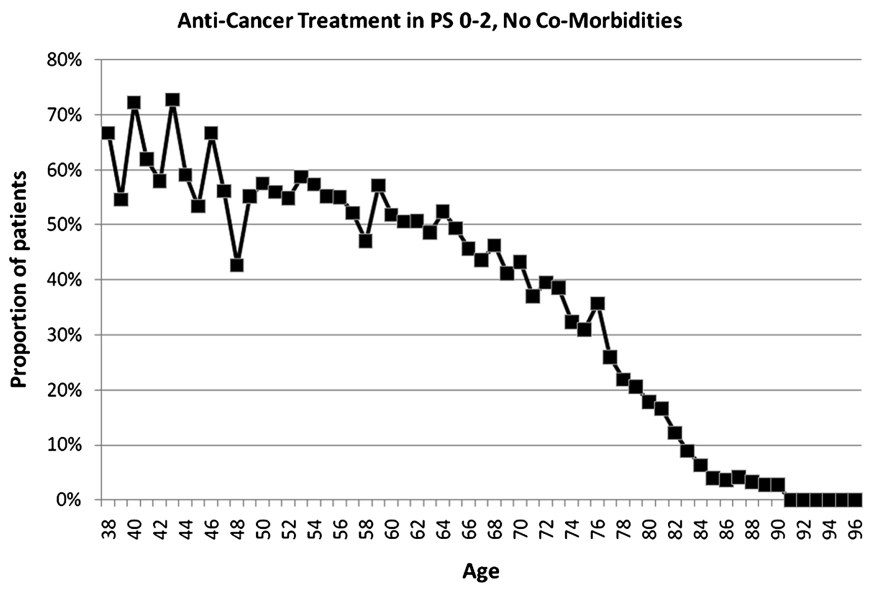

Abstract P215 Figure 1 Anti-Cancer treatment in PS 0-2, no comorbidities

Conclusions Age appears to be an important factor in management decisions in English lung cancer practice. The results indicate that further work is warranted to determine how far the results can be explained by patient preference, appropriate physician judgement and physician prejudice.

\section{P216 LUNG CANCER IN YOUNG PATIENTS: A RETROSPECTIVE STUDY}

doi:10.1136/thx.2010.151068.17

Rahuldeb Sarkar, Gareth Jones, Tania Peacock, Ana de Ramón Casado. Warrington Hospital, Warrington, UK

Introduction Over-representation of women, more advanced disease staging at presentation, better performance status and similar survival figures have been noticed in younger ( $<50$ years) lung cancer patients as compared to the general patient population with the disease.

Objective A retrospective study (December 2009) was done to derive clinicopathological data from young lung cancer patients treated in our hospital in the last 5 years. We also compared our findings with National Lung Cancer Audit (NLCA), 2007 in the UK. The NLCA data were considered as a reflector of the patients from all age groups.

Findings 28 patients were identified for the study. Median age was 48 years (37-50). 15(54\%) were female. $75 \%$ had WHO performance status (PS) 1 in patients with documented PS. $43 \%$ had family history of lung cancer. $89 \%$ were current smoker. Histology was achieved in $89 \%$. 68\% had non small cell carcinoma (NSCLC) including 39\% adeno carcinoma, $24 \%$ had small cell carcinoma; 2 had carcinoid. $55 \%$ of histology proven NSCLC had stage 4 disease at presentation. $86 \%$ had some form of treatment (surgery, chemotherapy or radiotherapy). 5(18\%) had resection. Survival related data are presented here:

\begin{tabular}{lll}
\hline & Median survival (days) & 1 year survival rate (\%) \\
\hline All patients & 254 & 32 \\
Male & 193 & 25 \\
Female & 261 & 36 \\
\hline
\end{tabular}

Comparison between our findings and the NLCA data are presented below [Abstract P216 Table 1].

\section{Abstract P216 Table 1}

\begin{tabular}{lll}
\hline & NLCA data & Local data \\
\hline Male:female & $1.4: 1$ & $0.86: 1$ \\
Histological confirmation & $68 \%$ & $89 \%$ \\
$\begin{array}{l}\text { Stage IV at presentation } \\
\text { in NSCLC }\end{array}$ & $32 \%$ & $55 \%$ \\
$\begin{array}{l}\text { Active treatment } \\
\text { received }\end{array}$ & $51 \%$ & $86 \%$ \\
$\begin{array}{l}\text { Resection in NSCLC } \\
\text { Median NSCLC survival }\end{array}$ & $14 \%$ & $17 \%$ \\
(day) & 232 & 246 \\
\hline
\end{tabular}

Conclusion Majority of these patients presented with advanced stage disease as in previously reported larger cohorts. Women were a majority but they had a better survival than men. $43 \%$ had positive family history suggesting a possible genetic factor. A good proportion in this patient group had favourable performance status resulting in higher resection rate and receiving of active treatment compared to overall patient population. However, this did not lead to better survival.

\section{P217 WHAT'S HAPPENING TO LUNG CANCER IN FEMALES?}

doi:10.1136/thx.2010.151068.18

A Ives, J Verne. South West Public Health Observatory, Bristol, UK

Aim To examine trends in female lung cancer incidence.

Introduction Lung cancer incidence in men has been decreasing for the past 2-3 decades, while for females it has increased. Furthermore, to achieve the national cancer mortality target reduction, it is essential that lung cancer incidence reduces as this is a major contributor to the mortality target. Mortality trends closely mirror that of incidence due to the poor prognosis of lung cancer.

Methodology The UK Association of Cancer Registries dataset was used to identify female lung cancer cases diagnosed between 1985 and 2006 in England and its constituent Regions. Three year rolling directly age-standardised rates (standardised to the European population) was calculated. National deprivation quintiles were used, where deprivation was based on the income domain of Indices of Multiple Deprivation 2007. The postcode of residence of each patient was used to assign the relevant deprivation quintile.

Results England lung cancer incidence for females significantly increased $(p<0.01)$ from 1985 to 1987 (32.3 per 100000) and 2004-2006 (35.4 per 100000$)$. Incidence and trends across Regions varies significantly. Further analysis at Network/LA level shows even more extreme variation in trends. By 2030, it is anticipated that lung cancer incidence will be similar for both males and females in the South West Region. In the past 20 years the inequalities gap for females is widening. Incidence rates have increased in the most deprived population $(30 \%)$ of the South West, while remaining relatively stable in the most affluent population of the region. Each Region shows different inequalities. 
Conclusion Smoking prevention is the key to reducing lung cancer. A lot of effort to encourage people to give up smoking has focused on men. Evidence of increasing female rates, more so in deprived areas; show that targeted efforts to support women need to be increased in order for large health gains to be achieved.

\section{P218 IS THERE ANY VALUE IN OBTAINING A TISSUE DIAGNOSIS IN SUSPECTED LUNG CANCER PATIENTS WITH A PERFORMANCE STATUS 3-4?}

doi:10.1136/thx.2010.151068.19

M Gautam, M Haris, S Huq, M Shaw, M Ledson, M Walshaw. Liverpool Heart and Chest Centre, Liverpool, UK

Introduction The National Lung Cancer Audit stipulates a minimum $75 \%$ tissue diagnosis rate in order to meet the standard required for Peer Review. Whilst this is imperative in patients suitable for potentially curative treatment $(\mathrm{PS}=1-2)$, in those with a poorer performance state ( $\mathrm{PS}=3-4)$ it has been suggested that investigations aimed at obtaining a tissue diagnosis lengthen the diagnostic process without improving the patient journey, thereby wasting resource and diminishing the quality of care. To look at this further, we studied patients with $\mathrm{PS}=3-4$ diagnosed at our large lung cancer unit (all-patients tissue diagnosis rate $>80 \%$ ), comparing their tissue diagnosis rate with treatment offered and ultimate outcome.

Methods All 108 patients (57 male) with PS=3-4 (18 PS=4) diagnosed at our unit during 2009 formed the study population (25\% of total diagnoses). Eighty-eight diagnostic procedures were attempted in 72 patients $(66 \%)$, and were successful in 59 of these (82\%) (15 small cell, 40 non-small cell, 4 other malignancies).

Results Although a tissue diagnosis was more likely to be attempted in those with $\mathrm{PS}=3$ than 4 ( $66 / 90$ vs $6 / 18, \chi^{2}=10.8, \mathrm{p}<0.01$ ), it was no more likely to be successful $\left(54 / 66\right.$ vs $\left.5 / 6, \chi^{2}=0.1, p=N S\right)$. Those with a tissue diagnosis were more likely to be offered chemo or radiotherapy (active treatment) $\left(28 / 59\right.$ vs $\left.5 / 49, \chi^{2}=17.5, p<0.001\right)$, with the remaining patients benefiting from best supportive care (BSC). Furthermore, Kaplan-Meier plots (censored at 150 days) demonstrated that those who underwent active treatment had an improved clinical outcome (median survival: treated group 81 days [IOR 48-143] vs BSC group 16 days [6-56], $\mathrm{p}<0.001$ ).

Conclusions This audit shows that patients with a poor performance state who undergo some form of active treatment gain a survival benefit. For clinical governance reasons, active treatment is unlikely to be offered to patients without a tissue diagnosis, underlining the need to appropriately investigate this patient group wherever possible. We achieved a tissue diagnosis rate of $55 \%$, and exhort other lung cancer units to adopt a similar approach to improve the clinical journey of these unfortunate and sometimes under treated patients.

\section{P219 IS THERE A ROLE FOR CONVENTIONAL TRANSBRONCHIAL NEEDLE ASPIRATION IN THE ENDOBRONCHIAL ULTRASOUND ERA?}

doi:10.1136/thx.2010.151068.20

U H Oltmanns, P Perera, M Slade. Papworth Hospital, Papworth Everard, Cambridge, UK

Introduction and Objectives It is believed by some that endobronchial ultrasound (EBUS) will render conventional transbronchial needle aspiration (cTBNA) obsolete. We sought to explore this question.

Methods Data on both conventional $(n=33)$ and a randomly selected equal number of EBUS-guided TBNA ( $n=33$ ), performed to biopsy mediastinal lymph nodes (LN), between August 2008 and May 2010, were extracted from a prospective database of bronchoscopy procedures. Measured variables were age, sex, sampled LN stations and sizes, sample results and final diagnoses for LN and underlying patient diagnosis.

Results Thirty-eight LN from 4 different LN stations in the cTBNA group and 46 LN from 5 different LN stations in the EBUS group were sampled. LN were significantly larger in the cTBNA group (median $2.65 \mathrm{~cm}$, range $0.90-7.00 \mathrm{~cm}$ ) compared to the EBUS-TBNA group (median $1.45 \mathrm{~cm}$, range $0.60-3.00 \mathrm{~cm} ; \mathrm{p}<0.001$ ). Accurate diagnoses were obtained in 29/33 (88\%) cTBNA cases and 26/33 $(79 \%)$ EBUS-TBNA ( $p=0.51$, Fisher's exact test). cTBNA confirmed 23 lung cancers, 1 lymphoma, 3 benign conditions and 2 true negative cases confirmed surgically. Four false negative LN samples were diagnosed with lung cancer $(n=3)$ and sarcoidosis $(n=1)$ by other methods. EBUS-TBNA provided an accurate diagnosis in 26 patients (lung cancer, $n=14$; other malignancy, $n=2$; benign cases, $\mathrm{n}=7$; true negative, $\mathrm{n}=3$ ). Five false negative cases were subsequently diagnosed with lymphoma $(n=1)$, lung cancer $(n=3)$ and other malignancy $(n=1)$. One non-diagnostic sample without further diagnostic clarification was considered false negative. A further sample with possible malignant cell contamination was considered false positive.

Discussion In a centre with access to both techniques, selected patients with mediastinal or hilar lymphadenopathy can be investigated with cTBNA without compromising diagnostic accuracy. Across the UK, cTBNA is potentially widely available, and requires minimal capital investment compared to EBUS-TBNA. We believe that cTBNA remains an attractive first-line diagnostic procedure for chest physicians.

\section{P220 HIGH PREVALENCE OF MALIGNANCY IN HIV INFECTED PATIENTS WITH ENLARGED MEDIASTINAL LYMPHADENOPATHY}

doi:10.1136/thx.2010.151068.21

${ }^{1} \mathrm{~J}$ Alçada, ${ }^{2} \mathrm{~N}$ Navani, ${ }^{3} \mathrm{M}$ Taylor, ${ }^{3} \mathrm{P}$ Shaw, ${ }^{4} \mathrm{R} \mathrm{F}$ Miller, ${ }^{2} \mathrm{~S} \mathrm{M}$ Janes. ${ }^{1}$ Department of HIV Medicine, Mortimer Market Centre, University College London Hospital, London, UK; ${ }^{2}$ Centre for Respiratory Research, University College London, London, UK; ${ }^{3}$ Department of Thoracic Radiology, University College London Hospital, London, UK ${ }^{4}$ Centre for Sexual Health and HIV Research, University College London, London, UK

Introduction Intra-thoracic lymphadenopathy is commonly observed in patients infected with HIV. The broad range of aetiologies and the non-specific clinical picture constitute a diagnostic challenge which has not been previously addressed. Therefore we aimed to identify the causes of mediastinal lymphadenopathy in HIV infected patients presenting to our specialist unit and to characterise their mode of diagnosis.

Methods We conducted a retrospective analytical cohort study of HIV positive patients who underwent CT scanning of the chest between January 2004 and December 2008. Each patient was followed clinically for at least 12 months.

Results 234 consecutive patients underwent chest CT in the study period. Employing the conventional CT cut-off for significant lymphadenopathy of $10 \mathrm{~mm}$ in short-axis, mediastinal lymphadenopathy was present in $49(21 \%)$ patients. In these 49 patients, the mean age was 40 (range 19-69) years. 43 patients were male and 24 of the male patients were homosexual. 25 (51\%) of patients were receiving antiretroviral therapy and 24 patients had a CD4 lymphocyte count below 250 cells $\times 10^{6} / 1$. A specific diagnosis was made in 42 cases. Lymphoma was the most common diagnosis with 9 cases, followed by TB with seven cases. Six patients had reactive lymphadenopathy secondary to pneumonia. There were four cases of Castleman's disease, four of malignancy and three of non- 